\title{
Identification of piRNAs in the central nervous system
}

\author{
EUN JOO LEE, ${ }^{1,3}$ SOURAV BANERJEE, ${ }^{1,3}$ HONGJUN ZHOU, ${ }^{1,3}$ ARUNA JAMMALAMADAKA, ${ }^{2}$ MARY ARCILA, ${ }^{1}$ \\ B.S. MANJUNATH, ${ }^{2}$ and KENNETH S. KOSIK ${ }^{1}$ \\ ${ }^{1}$ Neuroscience Research Institute and Department of Molecular, Cellular and Developmental Biology, University of California, Santa Barbara, \\ California 93106, USA \\ ${ }^{2}$ Electrical and Computer Engineering Department, University of California, Santa Barbara, California 93106, USA
}

\begin{abstract}
Piwi-interacting RNAs (piRNAs) are small noncoding RNAs generated by a conserved pathway. Their most widely studied function involves restricting transposable elements, particularly in the germline, where piRNAs are highly abundant. Increasingly, another set of piRNAs derived from intergenic regions appears to have a role in the regulation of mRNA from early embryos and gonads. We report a more widespread expression of a limited set of piRNAs and particularly focus on their expression in the hippocampus. Deep sequencing of extracted RNA from the mouse hippocampus revealed a set of small RNAs in the size range of piRNAs. These were confirmed by their presence in the piRNA database as well as coimmunoprecipitation with MIWI. Their expression was validated by Northern blot and in situ hybridization in cultured hippocampal neurons, where signal from one piRNA extended to the dendritic compartment. Antisense suppression of this piRNA suggested a role in spine morphogenesis. Possible targets include genes, which control spine shape by a distinctive mechanism in comparison to microRNAs.
\end{abstract}

Keywords: piRNA; deep sequencing; dendrite; spine development

\section{INTRODUCTION}

The discovery of piRNAs stemmed from a search for noncoding RNAs that interacted with the protein Piwi (Aravin et al. 2006; Girard et al. 2006; Grivna et al. 2006a; Watanabe et al. 2006). Piwi was identified as essential for Germ Line Stem Cell (GSC) maintenance in Drosophila (Lin and Spradling 1997) by mediating a highly conserved somatic signaling mechanism (Cox et al. 1998). Emerging from the class of piRNAs are two subtypes of small noncoding RNAs. One set is abundant in germ cells, appears to restrict transposable elements, and is generated by a mechanism called "ping-pong" (Aravin et al. 2007; Malone and Hannon 2009). A second set is derived from genomic regions depleted in transposons, is generated by an incompletely understood pathway called "primary processing," is present in somatic cells, and may have a role in the regulation of target mRNAs. The mouse genome encodes three Piwi-like proteins-MIWI, MILI, and MIWI2. MIWI is expressed after

\footnotetext{
${ }^{3}$ These authors contributed equally to this work.

Reprint requests to: Kenneth S. Kosik, Neuroscience Research Institute and Department of Molecular, Cellular and Developmental Biology, University of California, Santa Barbara, CA 93106, USA; e-mail: kosik@ lifesci.ucsb.edu; fax: (805) 893-2005.

Article published online ahead of print. Article and publication date are at http://www.rnajournal.org/cgi/doi/10.1261/rna.2565011.
}

birth in pachytene spermatocytes and spermatids and has been posited to act in translational control (Deng and Lin 2002). MILI and MIWI2 are the only Piwi-family proteins required for transposon silencing in fetal gonocytes (Aravin et al. 2008; Kuramochi-Miyagawa et al. 2008). MILI binds 26-nt piRNAs usually derived from sense strands of transposable element transcripts, and MIWI2 prefers 28-nt piRNAs derived from anti-sense transposable element transcripts (Aravin et al. 2008). These RNAs undergo ping-pong amplification, which consumes transposon transcripts during the generation of new small RNAs (Aravin et al. 2008).

By mass sequencing, more than 52,000 piRNAs were associated with MIWI, the mouse ortholog of Piwi, and more than 1000 piRNAs were associated with MILI, a Piwi family member (Aravin et al. 2006; Girard et al. 2006). The set of piRNAs falls in a size range of 24-31 bp and thus can be fractionated as a distinct pool from the smaller $\sim 21-$ nt miRNAs. The great majority of piRNAs comprise genomic clusters of 1 to $127 \mathrm{~kb}$ often within intergenic sequences. Although the genomic locations of clusters are conserved, there is very little conservation at the level of individual piRNA sequences (Aravin et al. 2006; Girard et al. 2006; Lau et al. 2006).

Primary piRNA processing has been reported in a Drosophila ovarian somatic cell line where Piwi, but not Aub or AGO3, is expressed (Lau et al. 2009; Saito et al. 2009). The 
genomic loci for these ovarian somatic cell piRNAs are flamenco and traffic jam ( $t j$ ), a large Maf gene (Saito et al. 2009). Another source of piRNAs is $3^{\prime}$-untranslated regions (UTRs) of specific genes in somatic cells of Drosophila ovaries, murine testis, and Xenopus eggs (Robine et al. 2009; Saito et al. 2009). A role for piRNAs in translational regulation has also been suggested particularly in settings in which transcription and translation are decoupled. MIWI associates with both piRNAs and mRNAs, particularly the capbinding complex, in cytosolic ribonucleoprotein and polysomal fractions (Grivna et al. 2006b). In this case, genes involved in spermiogenesis are transcribed during earlier stages of spermatogenesis and then translationally repressed for several days until their protein products are needed. The piRNA pathway is also involved in the decay of maternal mRNAs and translational repression in the early embryo. Specifically, the CCR4-mediated deadenylation of the mRNA encoding the embryonic posterior morphogen Nanos (Nos) depends on piRNAs complementary to a specific region in the nos $3^{\prime}$-untranslated region (Rouget et al. 2010). Reduced deadenylation when piRNA-induced regulation is impaired correlates with nos mRNA stabilization and translational derepression in the embryo, resulting in head development defects. Given the growing likelihood that piRNAs may have a role in translational regulation and the specialized role for local translation in neuronal dendrites, we used deep sequencing to reveal a set of piRNAs in the hippocampus.

\section{RESULTS}

\section{A limited set of piRNAs is relatively abundant in the hippocampus}

Deep sequencing small RNA libraries from male mouse hippocampus resulted in $14.83 \times 10^{6}$ total 35 -bp reads, and $66.7 \%$ of these reads were mapped to the mouse genome. A parallel experiment in the female mouse yielded $9.18 \times 10^{6}$ reads and results very similar to those reported below. Reads that aligned to rRNA, tRNA, and adaptor sequences (allowing two mismatches) were filtered. We further filtered the miRNAs based on reported miRNA precursor sequences (allowing two mismatches) and observed that $50.3 \%$ of the reads matched known miRNAs. The reads from these 35-bp runs were highly correlated with an independent sequencing run with 25-bp tags (data not shown).

After filtering, the remaining small RNAs ( $<24 \mathrm{nt}$ ) represented $29.7 \%$ of the total and could correspond to novel

C.
miRNAs, endogenous siRNAs, or other small RNAs. $11.3 \%$ of the small RNAs were $>24$ nt (Fig. 1A). These RNAs fell into a size range between 25 and 32 nt. Because Piwiassociated RNAs (piRNAs) fall in this size range, we assessed whether any of the small noncoding RNAs matched known piRNA sequences that are reported in the noncoding RNA database (RNAdb) and the piRNA bank (pirnabank.ibab. ac.in). We found that $0.76 \%$ of the total small RNA species matched known piRNA sequences. Of these, 172 piRNAs were detected with five or more tags and showed a size distribution between 24 and 32 nt (Fig. 1B). 59.3\% of these reads had a " $U$ " at position "1," suggesting bias toward " $U$ " at the $5^{\prime}$ end (Fig. 1C), and the base distribution for the first 10 nucleotides is shown (Supplemental Fig. 1). Also, they corresponded to the set reported by coimmunoprecipitation with MIWI. Most of the piRNAs identified in our sample corresponded to the set reported by coimmunoprecipitation with MIWI. The set of piRNAs most highly expressed are mapped to unique intergenic location (Table 1). Among 20 piRNAs, four piRNAs belong to a large cluster on chromosome 17 (DQ540285, DQ540284, DQ541506, DQ543676), which spanned $\sim 7000$ bp containing 19 known piRNAs in our sample. In addition to the known piRNAs, thousands of small RNA sequences of $>25$ nt were also found within the cluster. We validated the expression level of the six most
A.
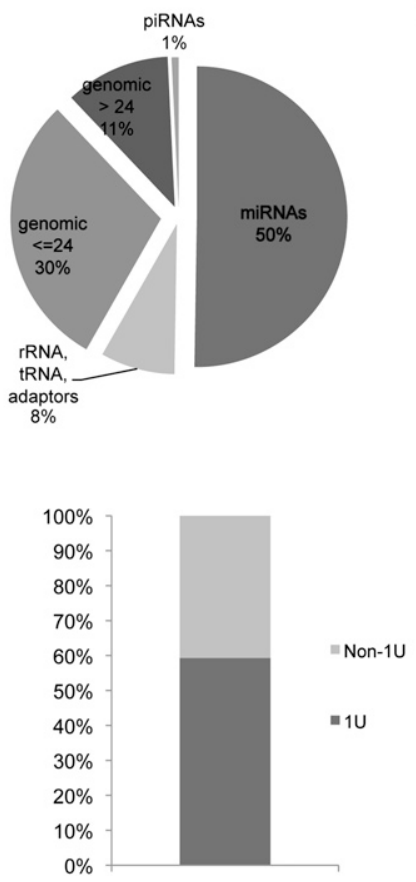

B.

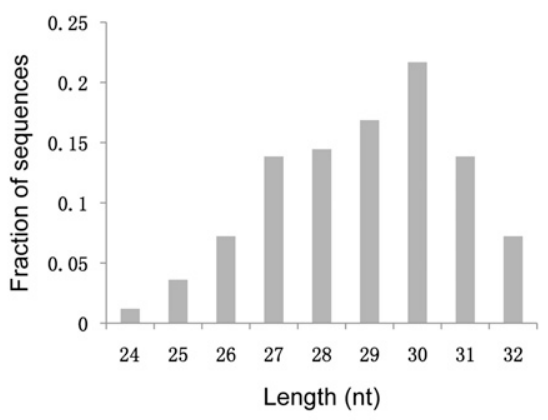

FIGURE 1. Sequencing mapping. (A) Small RNA mapping data. Approximately $50 \%$ of small RNA is mapped to miRNA; $\sim 30 \%$ of small RNA is $<24 \mathrm{nt}$, which could be novel miRNA, endogenous siRNA, or other small RNA; $\sim 11 \%$ of small RNA is mapped to sequences longer than $24 \mathrm{nt}$; and $\sim 1 \%$ is mapped to known piRNA sequences. (B) Size distribution of piRNAs in mouse hippocampus. piRNA with more than five tags are counted, and their size ranged between 25 and $32 \mathrm{nt}$. (C) Nucleotide bias of piRNA. Approximately $60 \%$ of piRNAs show that the first nucleotide is $\mathrm{U}$. 
TABLE 1. Abundant piRNAs in mouse hippocampus

\begin{tabular}{|c|c|c|c|}
\hline piRNA & Reads & Sequence & Genome location \\
\hline DQ541777 & 16130 & GGCTGGTCCGAAGGTAGTGAGTTATCTCAA & Chr6 (-): 47738150-47738179 \\
\hline DQ705026 & 6257 & CTGAAATGAAGAGAATACTCTTGCTGATC & Chr16 (+): 23109062-23109090 \\
\hline DQ555094 ${ }^{\mathrm{a}}$ & 3439 & TGGGGGGCCCAAGTCCTTCTGATCGAGGCCCA & Chr5 (+): 147072600-147072631 \\
\hline DQ719597 ${ }^{\mathrm{a}}$ & 2459 & GGTCGATGATGAGAGCTTTGTTCTGAGC & Chr4 (+): 130305590-130305617 \\
\hline DQ689086 & 1514 & TGCAATGATGTCATCTTACTACTGAAA & Chr19 (+): $8799064-8799090$ \\
\hline DQ540285 & 1433 & ATCGATGTGGTGCTCCGGAGTTCTCTTCGGGC & Chr17 (+): 39979944-39979975 \\
\hline DQ540981 & 1360 & CGGGCCGCCGGTGAAATACCACTACTCTCA & $\begin{array}{l}\text { Chr18 (-): 54858630-54858659 } \\
\text { Chr3 (+): 5860709-5860738 }\end{array}$ \\
\hline DQ720186 & 849 & AGAGCACCCCATTGGCTACCCAC & Chr7 (-): 108030822-108030844 \\
\hline DQ555093 & 775 & TGGGGGGCCCAAGTCCTTCTGATCGAGGC & Chr5 (+): 147072600-147072628 \\
\hline DQ540862 & 639 & CCGGGTGATGCGAATCGTAATCTGAGCCGA & Chr11 (+): 106362350-106362379 \\
\hline DQ540284 & 635 & ATCGATGTGGTGCTCCGGAGTTCTCTTCGGG & Chr17 (+): 39979944-39979974 \\
\hline DQ541506 & 580 & GATCGATGTGGTGCTCCGGAGTTCTCTT & Chr17 (+): 39979943-39979970 \\
\hline DQ539915 & 304 & АACATTTCCTGGGСCTTTCAGGAATACCACGA & Chr1 (-): 24622776-24622807 \\
\hline DQ540861 & 252 & CCGGGTGATGCGAATCGTAATCTGAGC & Chr11 (+): 106362350-106362376 \\
\hline DQ715526 & 207 & CACCAAGATGAGTGGTGCAAATCTGATC & Chr2 (-): 144091717-144091744 \\
\hline DQ543676 ${ }^{\mathrm{b}}$ & 182 & TCGATGTGGTGCTCCGGAGTTCTCTTCGGGC & Chr17 (+): 39979945-39979975 \\
\hline DQ722288 & 175 & TTACTTGATGATAGTAAAAGATCTGATG & Chr1 (+): 162968482-162968509 \\
\hline DQ551351 & 168 & TGCTTCAACAGTGCTTGAACGGAACCCGGT & Chr19 (+): 10057226-10057255 \\
\hline DQ550765 & 118 & TGCGGGATGCCTGGGTGACGCGATCTGCCCG & Chr15 (+): $82980194-82980224$ \\
\hline DQ708131 & 115 & TATCTGTGAGGATAAGTAACTCTGAGG & Chr19 (+): $8798524-8798550$ \\
\hline
\end{tabular}

abundant piRNAs (DQ541777, DQ705026, DQ555094, DQ719597, DQ689086, and DQ540285) by real-time PCR. All six of the tested piRNAs mapped to unique sites in the mouse genome. We detected these piRNAs in eight different mouse tissues including brain, hippocampus, heart, liver, lung, spleen, kidney, and testes (Fig. 2A). To assess the specificity of our real-time PCR reactions, we included a previously characterized germline piRNA (DQ715799) that was not expressed in the hippocampal small RNA library. Real-time PCR analysis showed that these piRNAs were expressed in all the tissue types tested with highly variable levels of expression (Supplemental Fig. 2). Among the set of piRNAs studied, brain, hippocampus, heart, and liver showed relatively high expression compared to kidney, spleen, lung, and testes (Fig. 2A). The piRNA (DQ541777) was predominantly expressed in mouse brain as well as in the hippocampus. Although these real-time PCR values validated the presence of those piRNAs tested, the correlation with the tag numbers by deep sequencing was not always precise (Supplemental Table 1).

To visualize the length of the piRNAs in our data set, we performed Northern blotting using total RNA fractions obtained from brain, hippocampus, heart, liver, lung, spleen, and testes. Northern blot data for DQ705026 showed that all somatic cell types expressed an $\sim 30$-nt-long piRNA transcript consistent with the 29-nt length of this piRNA by sequencing (Supplemental Fig. 3).

piRNAs are associated with Piwi proteins to form an effector multi-protein complex. In mouse, Piwi clade members are MIWI, MILI, and MIWI2. We looked for expression of these three Piwi members in the following murine tissues: whole brain, heart, liver, lung, kidney, and testes. MIWI protein was expressed in all tissue types tested but was lower than in testis (Supplemental Fig. 4). Expression of miwi mRNA was also observed by in situ hybridization in the Allen Brain Atlas in several brain regions, including hippocampus (Allen Brain Atlas, http://mouse. brain-map.org). These observations were confirmed by real-time PCR (data not shown). We could not detect expression of MILI and MIWI2 (data not shown). Therefore, MIWI is probably the critical Piwi member that forms a complex with somatic piRNAs in the nervous system. To test whether brain piRNAs are complexed with MIWI, we used a MIWI antibody (Girard et al. 2006) to coimmunoprecipitate those piRNAs identified in our sequencing experiment. Real-time PCR revealed that all of the top five uniquely expressed piRNAs (DQ541777, DQ705026, DQ719597, DQ689086, and DQ540285) were coimmunoprecipitated with MIWI (Fig. 2B; Supplemental Fig. 5).

\section{Subcellular localization of piRNP complexes in hippocampal neurons}

Although piRNAs are localized in the perinuclear region of germ cells (Malone et al. 2009), piRNAs from murine testis were reported present in the cytoplasm (Grivna et al. 2006b). These observations prompted us to test the neuronal 
A.

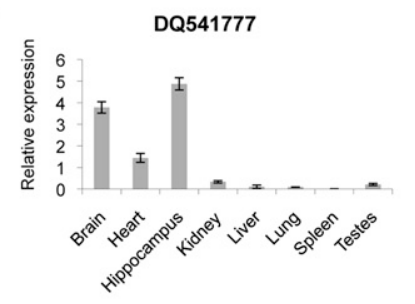

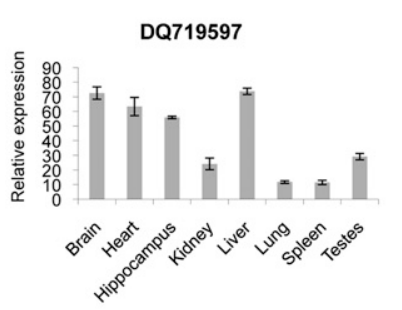

DQ715799

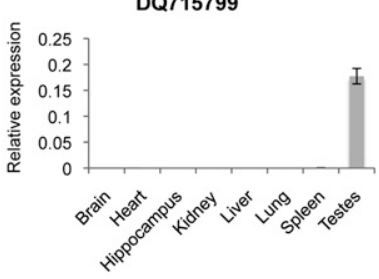

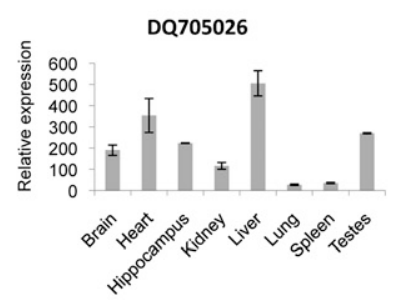
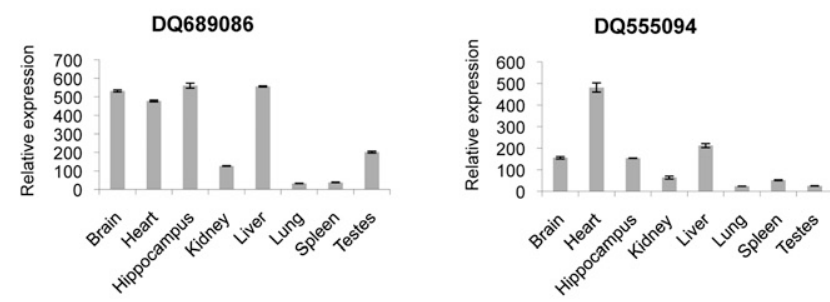

B.

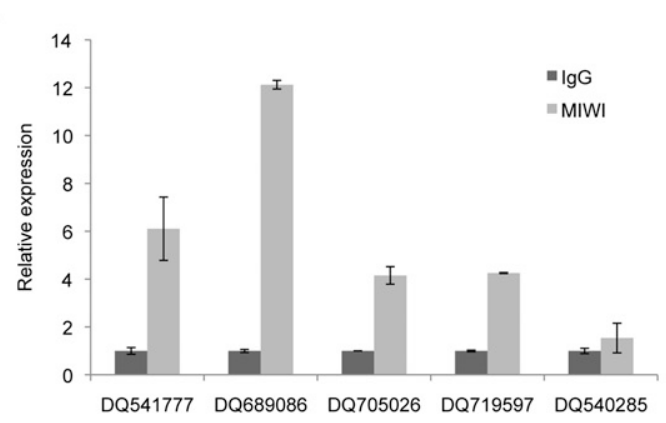

FIGURE 2. Analysis of piRNA expression and their association with piRNPs. (A) Real-time PCR expression in mouse tissues. Data were normalized by U6 small RNA and further multiplied by $10^{10}$ for the simplicity of the data presentation. DQ715799 is used as negative control. Amounts are represented as mean \pm SD. (B) Real-time PCR expression of piRNA after immunoprecipitation with MIWI antibody. Association of five piRNAs with MIWI was measured after immunoprecipitation by real-time PCR. Amounts of immunoprecipitated piRNAs (light gray) were normalized with IgG (dark gray). Amounts are represented as mean $\pm \mathrm{SD}$.

subcellular localization of four piRNAs (DQ541777, DQ705026, DQ719597, and DQ540285) by in situ hybridization using a digoxigenin (DIG) labeled locked nucleic acid (LNA)based anti-sense probe (Fig. 3; Supplemental Fig. 6). The specificity of the in situ hybridization was assessed by a sense probe as well as a universal control probe (Exiqon). The expression of these piRNAs was cytoplasmic in cultured mouse hippocampal neurons. Signal from one of the four piRNAs (DQ541777) extended throughout the dendrites in a punctate pattern typical of dendritic RNAs (Fig. 3A; Supplemental Fig. 6A).

The cytoplasmic localization of piRNAs in mouse hippocampal neurons prompted us to determine similarly the localization of MIWI, the predominantly expressed Piwi family protein. Immunostained cultured mouse hippocampal neurons revealed that MIWI is localized to cytoplasm in a punctate pattern (Fig. 3B; Supplemental Fig. 6B). MIWI puncta extended to neuronal dendrites as determined by colocalization with the dendritic marker MAP2 (Fig. 3C; Supplemental Fig. 6C). To support the functionality of piRNP complexes, we further showed that
piRNA and MIWI were colocalized in neuronal dendrites. To visualize piRNA in association with MIWI, we performed in situ hybridization using an LNA-based probe for piRNA (DQ541777) and then immunostained these neurons using antibody against MIWI (Fig. 3D). We observed that $50.15 \% \pm 17.23 \%$ of piRNA puncta colocalized with MIWI and $43.67 \% \pm 18.11 \%$ MIWI puncta colocalized with piRNA puncta. Taken together, these observations point to a role for the piRNP complex in the regulation of dendritic function.

\section{Neuronal function of piRNA DQ541777}

The localization of piRNA (DQ541777) in neuronal dendrite prompted us to design a loss-of-function experiment using the LNA-based antisense inhibitor of the specific piRNA (DQ541777) (Fig. 4). Our qPCR analysis showed that LNA-based antisense inhibition can significantly reduce the level of piRNA in rat hippocampal neuron $(P$-value $=0.00002)($ Supplemental Fig. 7$)$. We then cotransfected $\beta$-actin promoter-driven EGFP plasmid along 
with LNA-based antisense inhibitor of the piRNA (DQ541777) into hippocampal neurons at DIV7 and imaged these neurons at DIV21. We observed that inhibition of piRNA (DQ541777) activity led to a statistically significant decrease of dendrite spine area $(8.09 \% \pm 3.47 \%$, $P$-value $=0.0020883)$ (Fig. 4$)$. This observation suggests that piRNA (DQ541777) can function as a modulator of dendritic spine development.

These findings as well as the report showing a role for piRNA-target interactions in the mediation of Drosophila head development (Rouget et al. 2010) prompted us to identify putative targets of specific piRNAs. We bioinformatically searched candidate targets of five piRNAs (DQ541777, DQ705026, DQ555094, DQ719597, and DQ555093) with more than 500 tags. The piRNAtarget binding sites are based on sequence complementarity. Potential piRNA binding sites on mRNAs with known synaptic function were found using BLAST alignment. Short perfect alignments found by BLAST were manually extended to see if the remainder of the piRNA sequence matched the putative mRNA with partial complementarity. We also used RNAup (http://rna.tbi.univie.ac.at/cgi-bin/RNAup. cgi) to find the best binding sites with the minimum free energy. Our search revealed that piRNA DQ541777 could target the coding region of several neuronal mRNAs including Cdk5rap1 and Mark1/2 (Supplemental Fig. 8A-C). piRNAs DQ555093 and DQ555094 have putative binding sites in the coding region of AKAP 79/150 (also known as AKAP5, A-Kinase Anchoring Protein), a scaffolding protein localized to dendritic spines (Supplemental Fig. 8D; Robertson et al. 2009). A nonmuscle myosin, Myo5a (Myosin Va), is a putative target of piRNA DQ719597 (Supplemental Fig. 8E). Another interesting piRNA is DQ705026, which can presumably target Astrotactin, which has been implicated in neuronal migration (Supplemental Fig. 8F; Adams et al. 2002). Taken together, these observations suggest a novel gene regulatory control of nervous system function by piRNAs that has been previously shown to be restricted to the germline.
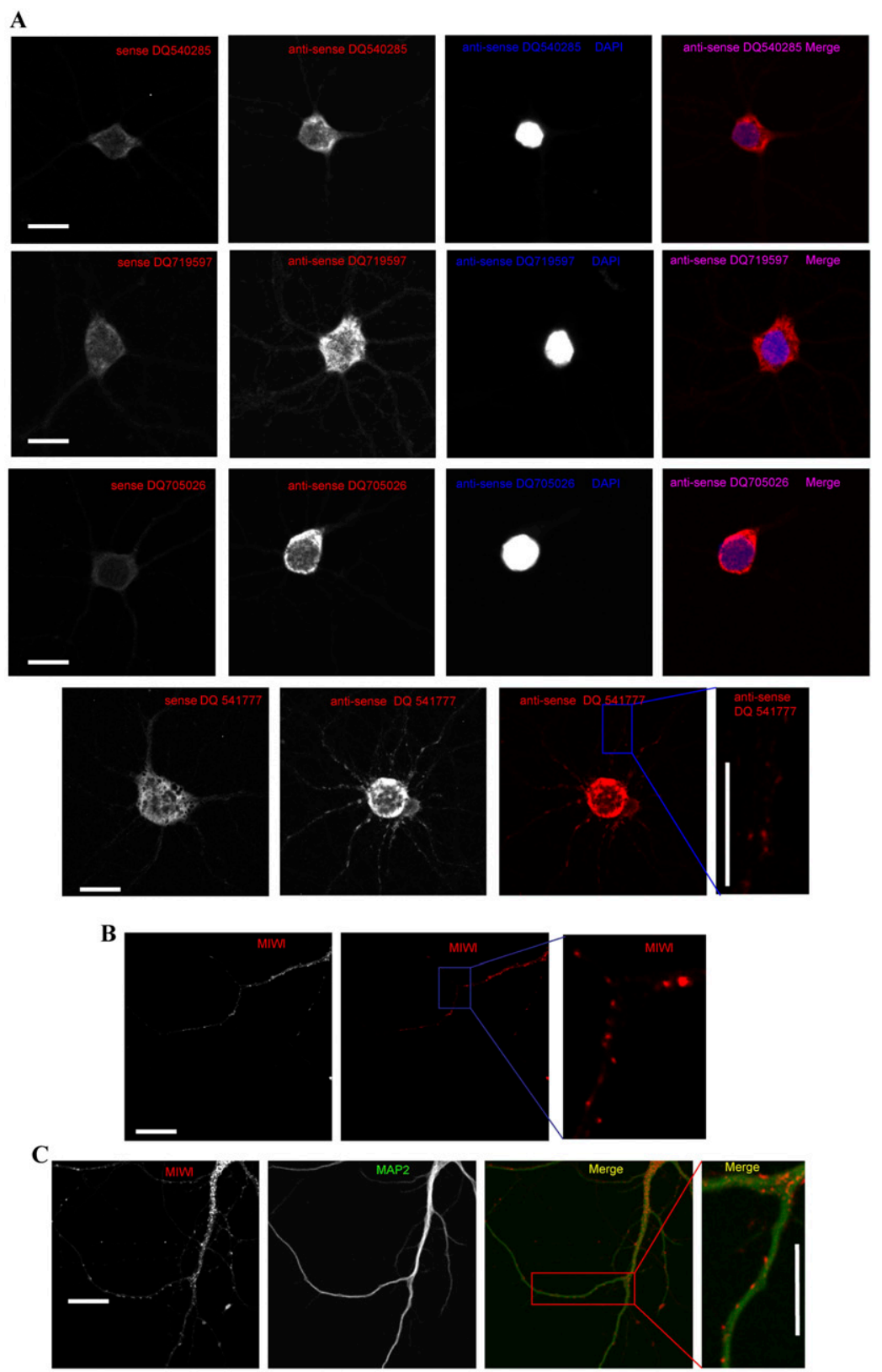

D
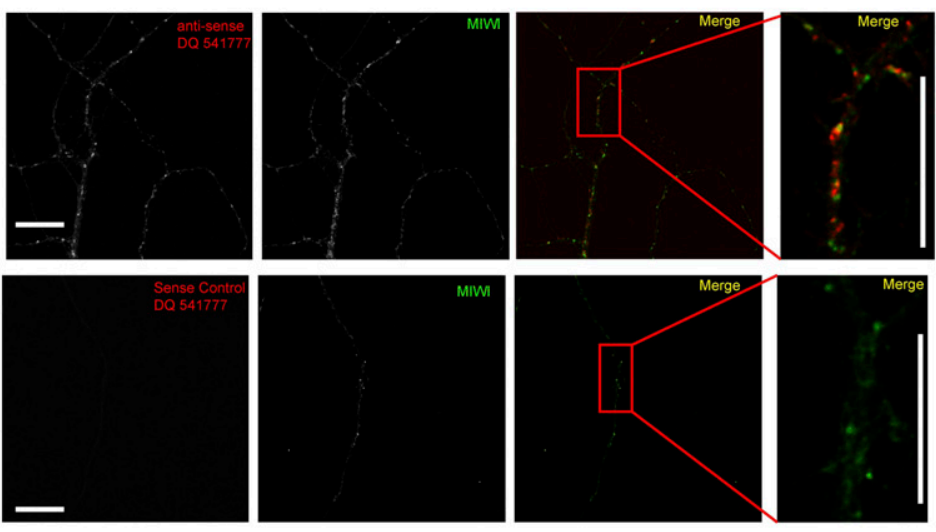

FIGURE 3. (Legend on next page) 


\section{DISCUSSION}

Deep sequencing the small RNAs in the mouse hippocampus revealed the presence of a limited set of piRNAs. However, our filtering for piRNAs required alignment with known piRNAs; therefore, the remaining $11 \%$ of small RNAs found in the 24-35-nt pool that align to the genome but have not been studied with regard to their association with PIWI proteins represents a larger candidate group of piRNAs in the hippocampus. The most highly expressed known piRNAs found in the hippocampus are intergenic with unique locations in the genome. The basis for classifying these small RNAs as piRNAs is the alignment of tags to sequences in the RNAdb or piRNA banks, validation of these tags by qPCR, the detection of these piRNAs in a MIWI IP, and the detection of these piRNAs by in situ hybridization.

piRNAs are most abundant and widely studied in germline or gonads. A single report described piRNAs in HeLa cells (Lu et al. 2010). Sharma et al. (2001) also reported that mRNA encoding the human homolog of the Drosophila piwi, hiwi, was most highly expressed in the testis followed by the kidney and then the brain. Our MIWI Western blot data also showed a similar rank ordering of expression (testis $>$ kidney $>$ brain).

The most highly expressed piRNA in the hippocampus was DQ541777. In contrast to the other piRNAs localized in neurons by in situ hybridization, the DQ541777 signal extended into the dendrites and had a punctate appearance typical of many RNA-containing compartments in dendrites. This localization is strongly suggestive of a role in regulating translation. Like other settings in which piRNAs may regulate translation, the dendrite is a compartment where translation is decoupled from transcription and instead is linked to synaptic stimulation. Often changes in translational regulation in dendrites are reflected in spine changes, and, indeed, antisense suppression of DQ541777 resulted in loss of spine area.

Several mRNAs scored highly as possible targets for hippocampal piRNAs. Among these were Cdk5rapl (also known as C42), which can function as an inhibitor of brainenriched kinase, Cdk5 (Ching et al. 2002). Interestingly, kinase activity of Cdk5 has already been implicated in the regulation of dendritic spine growth and shrinkage through modulation of cytoskeletal regulatory proteins, such as Rho GTPases (Cheung and Ip 2007; Fu and Ip 2007). Mark1/2 (Microtubule Affinity-Regulating Kinase 1/2) also scored highly as a putative piRNA target. Mark1/2 is an interacting partner of a key signaling protein, 14-3-3, that has been shown to regulate dendritic spine development (Angrand et al. 2006). Another putative target was AKAP79/150, which can function to regulate dendritic spine area (Robertson et al. 2009), similar to the observations here. Finally, Myosin Va is a potentially targeted motor protein enriched in the postsynaptic density (PSD), where it plays a critical role in the organization of postsynaptic density (Naisbitt et al. 2000).

The growing evidence that piRNAs have a role in somatic cells related to mRNA translation points to certain contrasts with the microRNA system. Both protein mechanisms that recognize these short RNAs are highly conserved; however, in contrast to miRNAs, which are among the most highly conserved transcripts, piRNAs are not conserved. One reason often given for the extraordinary conservation of miRNAs is the sequence constraints imposed by a large set of targets. The relative absence of conservation among piRNA sequences (Aravin et al. 2006; Girard et al. 2006; Lau et al. 2006) might suggest that their putative targets are more limited.

\section{MATERIALS AND METHODS}

\section{RNA preparation}

Six-week-old C57BL/6 male and female mice were killed, and mouse tissues were collected for total RNA isolation. Total RNA was isolated using the mirVana miRNA Isolation kit (Ambion) following the manufacturer's protocol. RNA from immunoprecipitation (IP) was isolated using Trizol (Invitrogen) and precipitated overnight. All RNAs were treated with DNase I (Ambion) for further experiments.

FIGURE 3. Localization of piRNAs and MIWI in cultured hippocampal neuron. (A) In situ hybridization of DIV21 mouse hippocampal neurons was performed with respective LNAbased sense and anti-sense probes as indicated. The nucleus was stained with DAPI; the merged image shows cytoplasmic localization of piRNAs. Although three piRNAs (DQ540285, DQ719597, and DQ705026) are restricted to the cell soma, piRNA (DQ541777) extended to the neuronal dendrite. In situ hybridization with sense control did not show hybridization signal. A portion of neuron with high-resolution image shown here is marked with a red box in Supplemental Figure 6. Scale bars, $20 \mu \mathrm{m}$. (B) Mouse hippocampal neurons at DIV21 were immunostained with an antibody against MIWI. Similar to RNA binding proteins, MIWI also showed a punctate pattern through the neuronal dendrite as well as the cell body. A portion of neuron with high-resolution image shown here is marked with a red box in Supplemental Figure 6. Scale bar, $20 \mu \mathrm{m}$. (C) Dendritic localization of piRNA (DQ 541777) was confirmed by immunostaining of mouse hippocampal neurons (DIV21) with a dendritic marker, MAP2. MIWI puncta colocalized with MAP2. A portion of neuron with high-resolution image shown here is marked with a red box in Supplemental Figure 6. Scale bars, $20 \mu \mathrm{m}$. (D) Association of piRNA and MIWI was analyzed by in situ hybridization of mouse hippocampal neurons (DIV21) using LNA-based anti-sense or sense probes and immunostained with a MIWI antibody. Both piRNA (DQ541777) and MIWI showed a punctate pattern in the neuronal dendrite. piRNA (DQ541777) and MIWI puncta showed colocalization throughout the neuronal dendrite. In situ hybridization with a sense control did not show any dendritic signal. A portion of neuron with high-resolution image shown here is marked with a red box in Supplemental Figure 6. Scale bars, $20 \mu \mathrm{m}$.

\section{Small RNA library preparation}

Ten micrograms of DNase I-treated total RNA was size-fractionated using the flashPAGE Fractionator (Ambion), and small RNA $\leq 40$ nt was collected. A small RNA library was prepared using the SOLiD Small RNA Expression Kit (Applied Biosystems). The quality of the library was assessed by the Agilent DNA 1000 chip (Agilent Technologies) and then quantified by quantitative PCR analysis using primers specific for both adaptors. We then used $280 \mathrm{pg}$ of library for emulsion PCR. The emulsion PCR was performed using the SOLiD ePCR system 3.0 (Applied Biosystems), and the library was sequenced by SOLiD sequencing. 
A
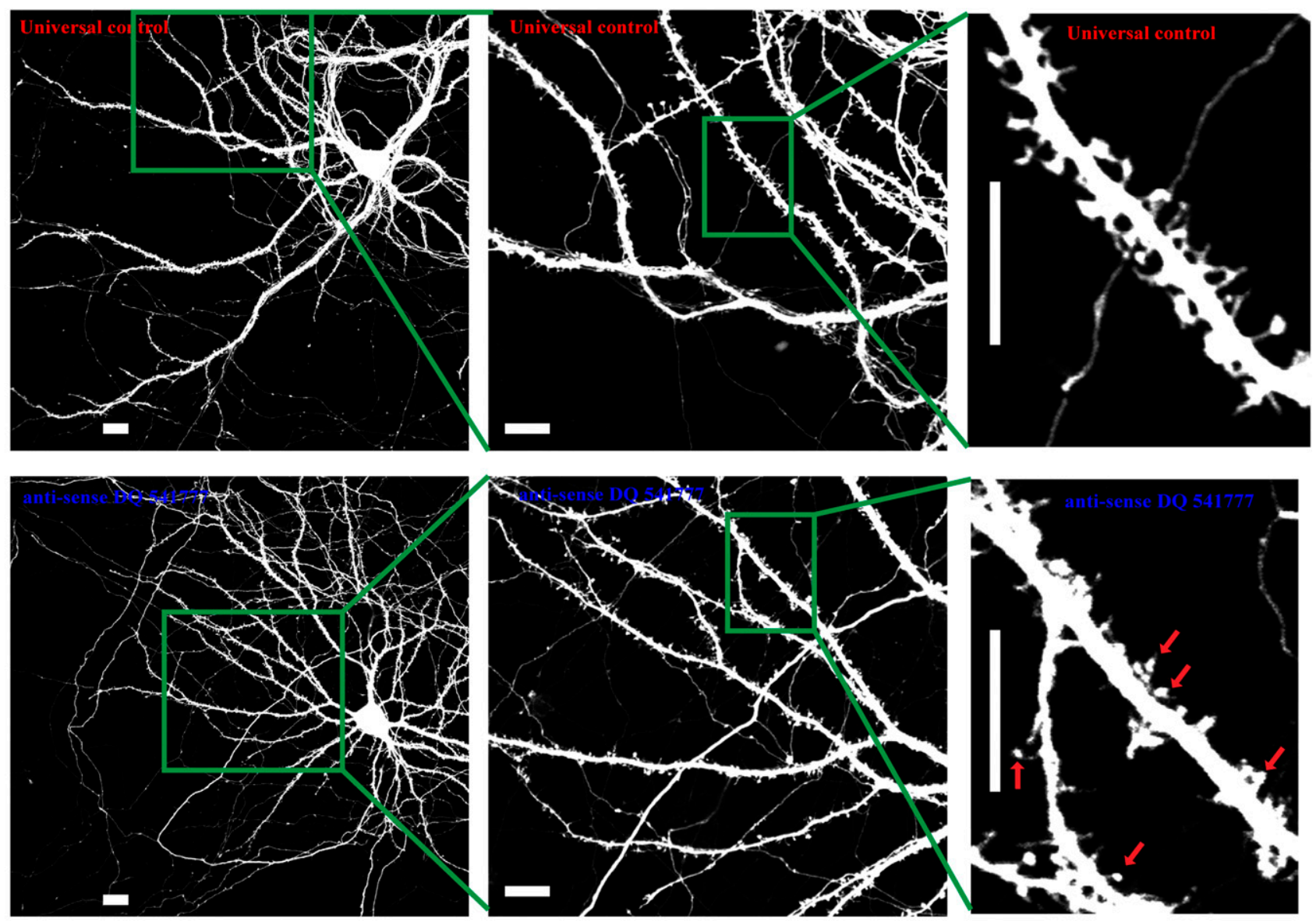

B

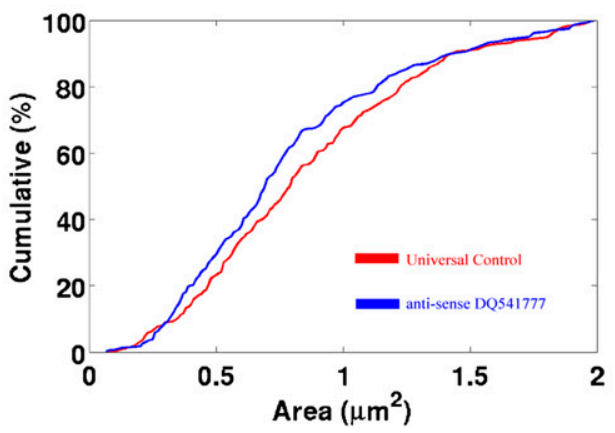

FIGURE 4. piRNA (DQ541777) regulates dendritic spine size. (A) Rat hippocampal neurons at DIV7 were co-transfected with either LNA-based inhibitor of piRNA (DQ541777) or universal control inhibitor along with EGFP plasmid. Neurons were then imaged at DIV21 and spine size was measured based on GFP fluorescence intensity. The dendrite area chosen for high-resolution images was marked with a green box. Scale bars, 20 $\mu \mathrm{m}$. (B) Quantitative analysis of mushroom spine area. More than 2075 spines (from 12 neurons) were analyzed in each condition to identify each spine type (mushroom, cup, stubby, and thin), and their respective area was measured. Change of spine size was observed only in mushroom spines. Mushroom spine area was calculated from more than 238 spines. The percentage change in area is represented as mean change. $P<0.05$ (KS test).

\section{Small RNA mapping analysis}

At a read length of $35 \mathrm{bp}$, all beads were analyzed in color space by the SOLiD small RNA Analysis pipeline (http://solidsoftwaretools. com). Briefly, the reads that have adaptors at the $5^{\prime}$ end or aligned with tRNAs and rRNAs were removed first. The reads were matched against miRNA precursors and the mouse genome. To determine the size of small RNAs, we first found the candidate alignments based on the first part of the reads and then extended the matching and compared the remaining part of the reads to adaptor sequences to identify the adaptors at the end of the reads.

For identification of the piRNAs in hippocampus, mouse piRNA sequences were downloaded from RNAdb (http://research.imb.uq. edu.au/rnadb/) and piRNA BANK (http://pirnabank.ibab.ac.in), 
which include the three largest data sets with thousands of distinct piRNAs expressed in mouse testes that bind MIWI or MILI (Aravin et al. 2006; Girard et al. 2006; Lau et al. 2006). All reads that matched any known miRNA precursors were filtered, and the remaining reads were mapped against the piRNA sequences. Only reads with $>25$-bp alignment were counted. To verify those matching on chromosomes, all reads were aligned to the genome based on the first $25 \mathrm{bp}$ and extended. Adaptors were always trimmed during extension. At most, two mismatches were allowed for mapping small RNA sequences to the genome. piRNA sequences were also aligned to the mouse genome by BLAT (Kent 2002), and mouse transposon annotations were downloaded from the UCSC Genome Browser (http:// genome.ucsc.edu/). The mouse tRNA sequences were downloaded from GtRNAdb (Chan and Lowe 2009), which contains tRNA gene predictions made by the tRNAscan-SE (Lowe and Eddy 1997). Mouse rRNA sequences were collected from BioMart (http:// www.biomart.org/).

\section{Real-time PCR of piRNA}

The Taqman small RNA assay for piRNA was custom designed by Applied Biosystems, and we followed their protocol with minor modifications described by Tang et al. (2008). DNase I-treated total RNA (100 ng) as well as immunoprecipitated RNA were reverse-transcribed using the high-capacity cDNA archive kit (Applied Biosystems) following the manufacturer's protocol. Quantitative PCR was normalized either with the small nucleolar RNA, U6, or with RNAs obtained through immunoprecipitation with control IgG. Real-time PCR was performed in duplicate using $1 \mu \mathrm{L}$ of a 1:20 dilution of cDNA. The normalized qPCR value was further multiplied by $10^{10}$ for simplicity of data presentation.

\section{Northern blotting}

Northern blotting was performed as described previously (Lau et al. 2001). Sixty micrograms of total RNA was separated on $15 \%$ of TBEUrea gel and transferred onto a Zeta-probe membrane (Bio-Rad). An LNA-based antisense probe for piRNA DQ705026 (Exiqon, Woburn, MA) was end-labeled with $\left[\gamma-{ }^{32} \mathrm{P}\right]$ ATP using the T4 polynucleotide kinase (Invitrogen) and used for hybridization.

\section{Neuronal culture}

Mouse as well as rat hippocampal neuron cultures were grown as described previously (Banerjee et al. 2009). Briefly, hippocampuses from embryonic day 15 (E15) embryos from timed-pregnant mice (C57BL/6) were dissected, and then dissociated neurons were plated (30 cells $\left./ \mathrm{mm}^{2}\right)$ onto poly-L-lysine $(1 \mathrm{mg} / \mathrm{mL})$ coated glass coverslips. Similarly, hippocampus from E18 embryos from timed-pregnant rats (Sprague-Dawley) were dissected and plated onto poly-L-lysine coated glass coverslips as described above. Neurons were co-cultured with mouse or rat glial cells accordingly in Neurobasal medium containing B27 supplement (Invitrogen) up to DIV21.

\section{Immunostaining}

DIV18-21 mouse hippocampal neurons were immunostained as described previously (Banerjee et al. 2009). Neurons were stained with antibodies against MIWI (1:300 dilution) and MAP2 (1:1000 dilution; Covance, Clone SMI-52). Immunostaining was visualized by cy5-conjugated anti-rabbit as well as cy3-conjugated anti- mouse secondary antibodies (Jackson Immunoresearch). Neurons were then imaged on an Olympus FV500 confocal microscope using an Olympus UPLFLN $40 \times \mathrm{NA}=1.3$ oil immersion objective. Illumination conditions were kept constant in all experiments.

\section{In situ hybridization}

In situ hybridization was performed as described previously (Banerjee et al. 2009) with minor modifications. Briefly, DIV1821 cultured hippocampal neurons were hybridized using digoxigenin-labeled (DIG Oligonucleotide Tailing Kit, second generation; Roche) Lock Nucleic Acid (LNA)-based antisense oligonucleotide (Exiqon) as well as sense oligonucleotide probes. Neurons were hybridized in hybridization mix (65\% formamide, $5 \times$ SSC, $0.1 \%$ Triton X-100, $9.2 \mathrm{mM}$ citric acid, $50 \mu \mathrm{g} / \mathrm{mL}$ heparin, $500 \mu \mathrm{g} / \mathrm{mL}$ tRNA, and $5 \mathrm{mM} \mathrm{MgCl}_{2}$ ) containing 3 pmol of DIG-labeled probes. Probe sequences are as follows: DQ541777 antisense, ACTCACTACCTTCGGACCAGCC; sense, CTGGTCCGAGGTAG TGAGTTA; DQ540285 antisense, GAGAACTCCGGAGCACCAC ATC; sense, GATGTGGTGCTCCGGAGTTCTC; DQ705026 antisense, TCAGCAAGAGTATTCTCTTCAT; sense, ATGAAGAGAA TACTCTTGCTGA; DQ719597 antisense, CAGAACAAAGCTCTC ATCATCG; sense, CGATGATGAGAGCTTTGTTCTG. Hybridization was performed overnight at $55^{\circ} \mathrm{C}$. After hybridization, neurons were washed as described in the whole-mount in situ protocol by Exiqon. Following the wash, neurons were incubated in blocking buffer (100 mM Tris- $\mathrm{HCl}$ at $\mathrm{pH} 7.4,150 \mathrm{mM} \mathrm{NaCl}$, and $1 \%$ proteasefree BSA fraction V) for $60 \mathrm{~min}$ at room temperature. Neurons were then incubated with cy3-labeled anti-DIG antibody (1:400 dilution; Jackson Immunoresearch) in blocking buffer overnight at $4^{\circ} \mathrm{C}$. After antibody incubation, neurons were washed three times in TBS (100 $\mathrm{mM}$ Tris- $\mathrm{HCl}$ at $\mathrm{pH} 7.4$ and $150 \mathrm{mM} \mathrm{NaCl}$ ) at room temperature with a 5-min interval between each wash. Neurons were visualized by confocal microscopy (FV500 microscope) using an Olympus UPLFLN $40 \times \mathrm{NA}=1.3$ oil immersion objective, and illumination conditions were kept constant in all experiments.

piRNA association with MIWI protein was assessed first by in situ hybridization using antisense as well as sense probes for piRNA (DQ541777) as described above and then immunostained with MIWI antibody (1:300 dilution).

\section{Immunoprecipitation and Western blot}

To identify components of the piRNA-associated protein complex, we immunoprecipitated piRNP complex from mouse (C57BL/6) brain lysate using antibodies against MIWI as described previously (Kiriakidou et al. 2005). The specificity of MIWI antibody was assessed as described previously (Girard et al. 2006). In brief, mouse hippocampus was homogenized in lysis buffer $(20 \mathrm{mM}$ Tris- $\mathrm{HCl}$ at $\mathrm{pH} 7.4,200 \mathrm{mM} \mathrm{NaCl}, 2.5 \mathrm{mM} \mathrm{MgCl}_{2}, 0.5 \% \mathrm{NP}-40$, $0.1 \%$ Triton $\mathrm{X}-100$, one tablet of EDTA-free protease inhibitors per $50 \mathrm{~mL}$ of buffer, and $0.1 \mathrm{U} / \mu \mathrm{L}$ RNasin). Then $30 \mu \mathrm{g}$ of MIWI and rabbit IgG antibodies was conjugated with Protein A agarose beads. Equal amounts of tissue lysates were incubated with Protein A agarose beads conjugated with antibodies for $4 \mathrm{~h}$ at $4^{\circ} \mathrm{C}$. Following incubation, beads were collected by centrifugation and washed five times with lysate buffer. The identity of proteins in immunoprecipitated complexes was analyzed by Western blot using half of the immunoprecipitated sample, and the other half of the immunoprecipitated sample was used to detect selected piRNA association with the immunoprecipitated complex by qPCR. 
Mouse tissue lysates $(40 \mu \mathrm{g})$ as well as immunoprecipitated protein complexes were resolved in $8 \%$ SDS-Polyacrylamide gel and then transferred onto PVDF membrane. Following transfer, the membrane was blotted using antibodies against MIWI, MILI, and MIWI2. Blots were then incubated with HRP conjugated antirabbit secondary antibody (Jackson Immunoresearch) and visualized by chemiluminescence (Pierce).

\section{Spine analysis}

Hippocampal neurons at DIV7 were cotransfected with LNA antisense piRNA inhibitor and EGFP plasmid (Addgene). Based on EGFP fluorescence, transfected neurons (DIV21) were imaged by confocal microscopy (FV500) using an Olympus UPLFLN $40 \times \mathrm{NA}=1.3$ oil immersion objective with illumination conditions kept constant in all experiments. Neurons were selected in a blinded manner under low resolution at which spines are not visible. Dendritic tracing, spine detection, and spine classification were performed with NeuronStudio (Rodriguez et al. 2008). A 4-class trainable classifier was created with 47 manually labeled examples. From the output spine text files, only the spine attachment points and center of mass points were used. From these we computed the angle of rotation necessary to make each spine upright and extracted a maximal region of interest around the spine. The cropped spine image was then fed into a dendritic reduction algorithm, which removes remnant pieces of the dendrite, after which a connected component analysis was performed to further isolate the spine of interest. Finally, the area was computed using a thresholded version of the remaining image. S/D ratio was calculated by the average intensity at the center of mass of the spine divided by the average intensity below the attachment point. The mean and standard deviation of the percentage change for spine measurements were found by re-sampling the data 100 times using the bootstrapping method. A 2-sided Kolmogorov-Smirnov test was used to determine $P$-values.

\section{SUPPLEMENTAL MATERIAL}

Supplemental material is available for this article.

\section{ACKNOWLEDGMENTS}

We thank Dr. Gregory J. Hannon (Cold Spring Harbor Laboratory) for MIWI, MILI, and MIWI2 antibodies; Snigdha Chatterjee for preparing hippocampal cultures; Christopher Douglas for synapse analysis; and the members of the Kosik laboratory for many generous discussions. The work was supported by the Hillblom Foundation and the Errett Fisher Foundation. E.J.L. is funded by a postdoctoral fellowship from the California Institute for Regenerative Medicine (CIRM). A.J. and B.S.M. are supported in part by an NSF award III0808772 .

\section{NOTE ADDED IN PROOF}

In a personal communication the Kandel lab has also found a piRNA in the nervous system.

Received November 30, 2010; accepted March 9, 2011.

\section{REFERENCES}

Adams NC, Tomoda T, Cooper M, Dietz G, Hatten ME. 2002. Mice that lack astrotactin have slowed neuronal migration. Development 129: $965-972$.

Angrand PO, Segura I, Volkel P, Ghidelli S, Terry R, Brajenovic M, Vintersten K, Klein R, Superti-Furga G, Drewes G, et al. 2006. Transgenic mouse proteomics identifies new 14-3-3-associated proteins involved in cytoskeletal rearrangements and cell signaling. Mol Cell Proteomics 5: 2211-2227.

Aravin A, Gaidatzis D, Pfeffer S, Lagos-Quintana M, Landgraf P, Iovino $\mathrm{N}$, Morris $\mathrm{P}$, Brownstein $\mathrm{MJ}$, Kuramochi-Miyagawa $\mathrm{S}$, Nakano T, et al. 2006. A novel class of small RNAs bind to MILI protein in mouse testes. Nature 442: 203-207.

Aravin AA, Hannon GJ, Brennecke J. 2007. The Piwi-piRNA pathway provides an adaptive defense in the transposon arms race. Science 318: 761-764.

Aravin AA, Sachidanandam R, Bourc'his D, Schaefer C, Pezic D, Toth KF, Bestor T, Hannon GJ. 2008. A piRNA pathway primed by individual transposons is linked to de novo DNA methylation in mice. Mol Cell 31: 785-799.

Banerjee S, Neveu P, Kosik KS. 2009. A coordinated local translational control point at the synapse involving relief from silencing and MOV10 degradation. Neuron 64: 871-884.

Chan PP, Lowe TM. 2009. GtRNAdb: a database of transfer RNA genes detected in genomic sequence. Nucleic Acids Res 37: D93D97.

Cheung ZH, Ip NY. 2007. The roles of cyclin-dependent kinase 5 in dendrite and synapse development. Biotechnol J 2: 949-957.

Ching YP, Pang AS, Lam WH, Qi RZ, Wang JH. 2002. Identification of a neuronal Cdk5 activator-binding protein as Cdk5 inhibitor. J Biol Chem 277: 15237-15240.

Cox DN, Chao A, Baker J, Chang L, Qiao D, Lin H. 1998. A novel class of evolutionarily conserved genes defined by piwi are essential for stem cell self-renewal. Genes Dev 12: 3715-3727.

Deng W, Lin H. 2002. miwi, a murine homolog of piwi, encodes a cytoplasmic protein essential for spermatogenesis. Dev Cell 2: 819-830.

Fu AK, Ip NY. 2007. Cyclin-dependent kinase 5 links extracellular cues to actin cytoskeleton during dendritic spine development. Cell Adh Migr 1: 110-112.

Girard A, Sachidanandam R, Hannon GJ, Carmell MA. 2006. A germline-specific class of small RNAs binds mammalian Piwi proteins. Nature 442: 199-202.

Grivna ST, Beyret E, Wang Z, Lin H. 2006a. A novel class of small RNAs in mouse spermatogenic cells. Genes Dev 20: 1709-1714.

Grivna ST, Pyhtila B, Lin H. 2006b. MIWI associates with translational machinery and PIWI-interacting RNAs (piRNAs) in regulating spermatogenesis. Proc Natl Acad Sci 103: 1341513420.

Kent WJ. 2002. BLAT-the BLAST-like alignment tool. Genome Res 12: $656-664$

Kiriakidou M, Nelson P, Lamprinaki S, Sharma A, Mourelatos Z. 2005. Detection of microRNAs and assays to monitor microRNA activities in vivo and in vitro. Methods Mol Biol 309: 295-310.

Kuramochi-Miyagawa S, Watanabe T, Gotoh K, Totoki Y, Toyoda A, Ikawa M, Asada N, Kojima K, Yamaguchi Y, Ijiri TW, et al. 2008. DNA methylation of retrotransposon genes is regulated by Piwi family members MILI and MIWI2 in murine fetal testes. Genes Dev 22: 908-917.

Lau NC, Lim LP, Weinstein EG, Bartel DP. 2001. An abundant class of tiny RNAs with probable regulatory roles in Caenorhabditis elegans. Science 294: 858-862.

Lau NC, Seto AG, Kim J, Kuramochi-Miyagawa S, Nakano T, Bartel DP, Kingston RE. 2006. Characterization of the piRNA complex from rat testes. Science 313: 363-367.

Lau NC, Robine N, Martin R, Chung WJ, Niki Y, Berezikov E, Lai EC. 2009. Abundant primary piRNAs, endo-siRNAs, and microRNAs in a Drosophila ovary cell line. Genome Res 19: 1776-1785. 
Lin H, Spradling AC. 1997. A novel group of pumilio mutations affects the asymmetric division of germline stem cells in the Drosophila ovary. Development 124: 2463-2476.

Lowe TM, Eddy SR. 1997. tRNAscan-SE: a program for improved detection of transfer RNA genes in genomic sequence. Nucleic Acids Res 25: 955-964.

Lu Y, Li C, Zhang K, Sun H, Tao D, Liu Y, Zhang S, Ma Y. 2010. Identification of piRNAs in HeLa cells by massive parallel sequencing. BMB Rep 43: 635-641.

Malone CD, Hannon GJ. 2009. Small RNAs as guardians of the genome. Cell 136: 656-668.

Malone CD, Brennecke J, Dus M, Stark A, McCombie WR, Sachidanandam R, Hannon GJ. 2009. Specialized piRNA pathways act in germline and somatic tissues of the Drosophila ovary. Cell 137: 522-535.

Naisbitt S, Valtschanoff J, Allison DW, Sala C, Kim E, Craig AM, Weinberg RJ, Sheng M. 2000. Interaction of the postsynaptic density-95/guanylate kinase domain-associated protein complex with a light chain of myosin-V and dynein. J Neurosci 20: 4524-4534.

Robertson HR, Gibson ES, Benke TA, Dell'Acqua ML. 2009. Regulation of postsynaptic structure and function by an A-kinase anchoring protein-membrane-associated guanylate kinase scaffolding complex. J Neurosci 29: 7929-7943.

Robine N, Lau NC, Balla S, Jin Z, Okamura K, Kuramochi-Miyagawa S, Blower MD, Lai EC. 2009. A broadly conserved pathway generates 3'UTR-directed primary piRNAs. Curr Biol 19: 2066-2076.
Rodriguez A, Ehlenberger DB, Dickstein DL, Hof PR, Wearne SL. 2008. Automated three-dimensional detection and shape classification of dendritic spines from fluorescence microscopy images. PLoS ONE 3: e1997. doi: 10.1371/journal. pone.0001997.

Rouget C, Papin C, Boureux A, Meunier AC, Franco B, Robine N, Lai EC, Pelisson A, Simonelig M. 2010. Maternal mRNA deadenylation and decay by the piRNA pathway in the early Drosophila embryo. Nature 467: 1128-1132.

Saito K, Inagaki S, Mituyama T, Kawamura Y, Ono Y, Sakota E, Kotani H, Asai K, Siomi H, Siomi MC. 2009. A regulatory circuit for piwi by the large Maf gene traffic jam in Drosophila. Nature 461: 1296-1299.

Sharma AK, Nelson MC, Brandt JE, Wessman M, Mahmud N, Weller KP, Hoffman R. 2001. Human CD34(+) stem cells express the hiwi gene, a human homologue of the Drosophila gene piwi. Blood 97: 426-434.

Tang F, Hayashi K, Kaneda M, Lao K, Surani MA. 2008. A sensitive multiplex assay for piRNA expression. Biochem Biophys Res Commun 369: 1190-1194.

Watanabe T, Takeda A, Tsukiyama T, Mise K, Okuno T, Sasaki H, Minami N, Imai H. 2006. Identification and characterization of two novel classes of small RNAs in the mouse germline: retrotransposon-derived siRNAs in oocytes and germline small RNAs in testes. Genes Dev 20: 1732-1743. 

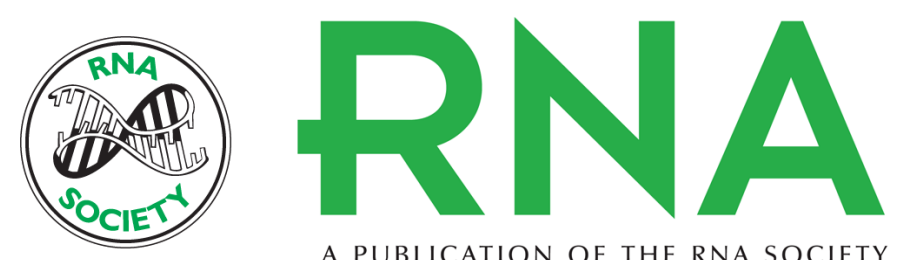

A PUBLICATION OF THE RNA SOCIETY

\section{Identification of piRNAs in the central nervous system}

Eun Joo Lee, Sourav Banerjee, Hongjun Zhou, et al.

RNA 2011 17: 1090-1099 originally published online April 22, 2011

Access the most recent version at doi:10.1261/rna.2565011

Supplemental
Material http://rnajournal.cshlp.org/content/suppl/2011/03/28/rna.2565011.DC1

References This article cites 35 articles, 16 of which can be accessed free at: http://rnajournal.cshlp.org/content/17/6/1090.full.html\#ref-list-1

License

Email Alerting Receive free email alerts when new articles cite this article - sign up in the box at the Service top right corner of the article or click here.

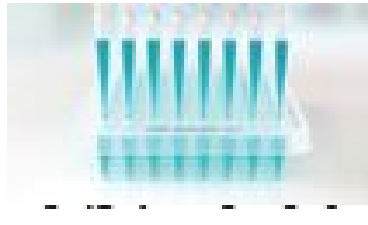

\section{Providing Precise Solutions for} your research.

To subscribe to RNA go to:

http://rnajournal.cshlp.org/subscriptions 